\title{
UNIVERSAL SPIN-HALL CONDUCTANCE FLUCTUATIONS IN TWO-DIMENSIONAL MESOSCOPIC SYSTEMS
}

\author{
ZHENHUA QIAO \\ Department of Physics, The University of Texas, Austin, TX 78712, USA \\ zhqiao@physics.utexas.edu \\ WEI REN \\ Department of Physics, The University of Arkansas, Fayetteville, AR 72701, USA \\ weiren@uark.edu

\section{JIAN WANG} \\ Department of Physics and the Center of Theoretical and Computational Physics, \\ The University of Hong Kong, Hong Kong, China \\ jianwang@hkusua.hku.hk
}

Received 20 December 2010

\begin{abstract}
The mesoscopic transport physics of spin Hall effect and quantized spin Hall effect is briefly reviewed. This paper concentrates on the universal spin Hall conductance fluctuations discovered in both effects. We present various model Hamiltonians corresponding to different physical problems arising recently in condensed matter physics, ranging from two-dimensional electron gas to topological insulators. Green's function formalism within a tight-binding model and the random matrix theory are introduced to study the same electron transport mechanism. The calculated spin Hall conductance results are discussed and some excellent agreement has been found from different approaches. A short summary and our perspectives are provided at the end of paper.
\end{abstract}

Keywords: Spin Hall effect; quantum spin Hall effect; spin-orbit coupling.

\section{Introduction}

Understanding and manipulating electronic currents of charge and spin are the major goals of the emerging research field of spintronics. ${ }^{1}$ Considerable attention has focused on the spin Hall effect ${ }^{2-6}$ and quantum spin-Hall effect, ${ }^{7-9}$ which provide mechanisms for generating spin current or spin accumulation thanks to the coupling between spin and orbital degrees of freedom. In analogy to the famous classic Hall effect, an applied electric current on the spin-orbit coupled systems can induce sidewise movement of pure spin currents $I_{s}^{U, D}=(\hbar / 2)\left(I^{\uparrow}-I^{\downarrow}\right)=G_{s H}\left(V_{L}-V_{R}\right)$ schematically shown in Fig. 1. As a result, the spin-up and spin-down carriers are deflected to the opposite edges of the sample. Moreover, spin Hall effect also offers a semiconductor analog of the Stern-Gerlach device to spatially separate up and down 


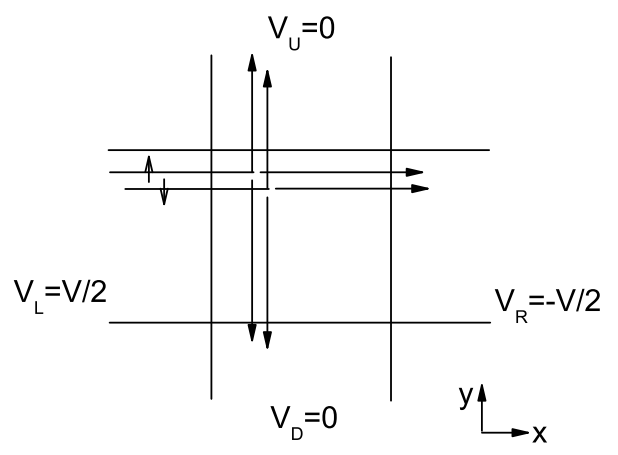

Fig. 1. Four-terminal setup of the Hall-bar device with four semi-infinite electrodes serving as electron reservoirs with different chemical potentials. Spin-orbit coupling only exists in the central scattering region, left and right leads. The longitudinal bias $V$ induces a charge current from left to right electrodes, while between the up and down electrodes there exists simultaneously non-zero spin current and zero charge current.

spin electrons. It is worth mentioning that such an effect is electrically controllable by gate voltages, without applying magnetic fields or using ferromagnet materials. The quantized version of the spin-Hall effect, also named as quantum spin-Hall insulator, is a recently discovered topological state in the massive Dirac fermion systems. This insulator resembles ordinary insulator in the bulk, while it shows topologically protected helical edge states at the boundary when the Fermi energy lies within the energy gap between the valence and conduction bands. For a given energy in the gap, the available electronic states at the same boundary possess opposite spins, so scattering is strongly suppressed and conduction on the surface is dissipationless. ${ }^{7,8,10,11}$

Universal conductance fluctuation is a reproducible and time-independent fingerprint of a given mesoscopic sample. ${ }^{12,13}$ The fluctuation pattern has a magnitude of the electric conductance quantum $e^{2} / h$ regardless of the averaged quantity. It was found and extensively investigated since the $1980 \mathrm{~s}$, and has been a hallmark of wave nature of electron. About twenty years after the first discovery of the universal conductance fluctuation, this fundamental concept has been successfully extended to the spin transport regime. In this brief review, we will discuss the universal spin Hall conductance fluctuation (USCF) ${ }^{14,15}$ and universal quantized spin Hall conductance fluctuation. ${ }^{16}$

In order to investigate the intriguing conductance characteristics of a mesoscopic system in the presence of disorders, it is imperative to carry out statistical studies over sufficient ensembles of samples. It is well-known that the symmetries play determining roles in the underlying physics. These universality classes include ${ }^{17,18}$ : circular orthogonal ensemble (COE) characterized by symmetry index $\beta=1$ when the time-reversal and spin-rotation symmetries are present; circular unitary ensemble (CUE) $\beta=2$ if time-reversal symmetry is broken; and circular symplectic ensemble (CSE) $\beta=4$ if the spin-rotation symmetry is broken while time-reversal 
symmetry is preserved. Here the symmetry index $\beta=1,2$ and 4 counts the number of degrees of freedom in the Hamiltonian matrix elements. Another notable factor is the dimensionality of the mesoscopic systems under study. Zero, one and two dimensional diffusive regimes give root mean square of conductance $c_{d} e^{2} / h \sqrt{\beta}$ with $c_{d}=0.70,0.73,0.86$ respectively. The universal conductance fluctuation, based on the perturbation theory method, ${ }^{12}$ was first found to be only weakly dependent on the dimensionality, which were later confirmed by the random matrix theory. ${ }^{17}$ This discovery brought a revolution in our belief of the electrons wave property in quantum physics. The electric conductance or transmission probability depends sensitively on the interference of electrons propagating through the diffusive samples. The motion of a single scattering center in the impurity configuration can induce change of the conductance of order $e^{2} / h$ by accumulating sufficient phase shift. ${ }^{19}$ Therefore, the universal conductance fluctuation is one of the most significant signatures in condensed matter physics. With another exciting technology spintronics developed since the 1980s, important universalities of such have just recently been revealed in the spin Hall effect arising from spin-orbit interaction as well.

The paper is organized as follows. In Sec. 1.1, we briefly introduce the Hamiltonian models of the conventional and quantized spin-Hall effects, followed by the theoretical methods. In Sec. 2, the USCF of the conventional spin-Hall effect is discussed, and a Random Matrix Theory confirmation of it is also included. In Sec. 3, the universal quantized spin-Hall conductance fluctuation $\left(\mathrm{USCF}_{2}\right)$ of the quantized spin-Hall effect is discussed. Finally, short summary and prospectives are given in Sec. 4.

\subsection{Theoretical models and methods}

\subsubsection{Two-dimensional spin-orbit coupling lattice model}

We consider a two-dimensional electron gas with spin-orbit coupling of Rashba or/and Dresselhaus types. The generalized single-particle Hamiltonian can be written as $^{20,21}$ :

$$
\begin{aligned}
H= & \frac{\left[\mathbf{p}+\frac{e}{c} \mathbf{A}\right]^{2}}{2 m^{*}}+V+\mu \mathbf{B} \cdot \sigma+\frac{\alpha_{S O}}{\hbar}\left[\sigma \times\left(\mathbf{p}+\frac{e}{c} \mathbf{A}\right)\right]_{z} \\
= & \frac{1}{2 m^{*}}\left[-\hbar^{2} \nabla^{2}+i \hbar \frac{e}{c} \nabla \cdot \mathbf{A}+i \hbar \frac{e}{c} \mathbf{A} \cdot \nabla+\frac{e^{2}}{c^{2}} A^{2}\right]+V \\
& +\mu \mathbf{B} \cdot \sigma+\frac{\alpha_{S O}}{\hbar}\left[-i \hbar \nabla_{y} \sigma_{x}+i \hbar \nabla_{x} \sigma_{y}+\sigma_{x} \frac{e}{c} A_{y}-\sigma_{y} \frac{e}{c} A_{x}\right]
\end{aligned}
$$

where $p$ is the momentum, $m^{*}$ is the effective electron mass, $\sigma$ are Pauli matrices, $V$ is the confining potential, $\mu$ is the magnetic moment, $\alpha_{S O}$ is the strength of spin-orbit coupling, and the vector potential can be expressed as $\mathbf{A}=(-B y, 0,0)$ under the Landau gauge. The two-terminal charge conductance is calculated 
using the Landauer-Büttiker formula $G=\left(2 e^{2} / h\right) T(E)$, where the transmission coefficient $T$ is given by $T(E)=\operatorname{Tr}\left[\Gamma_{L} G_{C}^{r} \Gamma_{R} G_{C}^{a}\right]$, and 2 arises from the spin degeneracy. Here, quantities $\Gamma_{L, R}=i\left(\Sigma_{L, R}^{r}(E)-\Sigma_{L, R}^{a}(E)\right)$ are the line-width functions describing the coupling between the left/right lead and scattering region, and can be obtained by calculating the self-energies $\Sigma_{L, R}^{r}$ due to semi-infinite leads using a recursive method. ${ }^{22} G_{C}^{r}$ and $G_{C}^{a}$ are the retarded and advanced Green's functions of the central disordered region. The conductance fluctuation is defined as $\operatorname{rms}(G)=\sqrt{\left\langle G^{2}\right\rangle-\langle G\rangle^{2}}$, where $\langle\cdots\rangle$ denotes averaging over an ensemble of samples with different disorder configurations of the same disorder strength. In the presence of Rashba spin-orbit coupling, the tight-binding Hamiltonian is written $\operatorname{as}^{14,23,24}$ :

$$
\begin{aligned}
H= & -t \sum_{i j \sigma} c_{i, \sigma}^{\dagger} c_{j, \sigma}+\sum_{i, \sigma} \varepsilon_{i} c_{i \sigma}^{\dagger} c_{i \sigma}+t_{S O} \sum_{i}\left[\left(c_{i, \uparrow}^{\dagger} c_{i+x, \downarrow}-c_{i, \downarrow}^{\dagger} c_{i+x, \uparrow}\right)\right. \\
& \left.-i\left(c_{i, \uparrow}^{\dagger} c_{i+y, \downarrow}+c_{i, \downarrow}^{\dagger} c_{i+y, \uparrow}\right)+\text { h.c. }\right]
\end{aligned}
$$

where $c_{i, \sigma}^{\dagger}$ is the creation operator for an electron with spin $\sigma$ on site $i$, and $x$ and $y$ are unit vectors along $x$ and $y$ directions. Here $t=\hbar^{2} / 2 m a^{2}$ is the nearest neighbor hopping energy and $t_{S O}=\alpha_{S O} / 2 a$ is the Rashba spin-orbit coupling with the square lattice constant $a$ being unity. The on-site energy is given by $\varepsilon_{i}=4 t$. We apply static Anderson-type disorder to the on-site energy with a uniform distribution in the interval $[-W / 2, W / 2]$, where $W$ measures the nonmagnetic disorder strength.

The spin Hall conductance $G_{s H}$ (see upper inset of Fig. 2 for the setup) is calculated using the Landauer-Büttiker formula ${ }^{25}$ :

$$
G_{s H}=\frac{e}{8 \pi}\left[\left(T_{\uparrow 2,1}-T_{\downarrow 2,1}\right)-\left(T_{\uparrow 2,3}-T_{\downarrow 2,3}\right)\right]
$$

where the spin-resolved transmission coefficient is given by $T_{2 \sigma, 1}=\operatorname{Tr}\left(\Gamma_{2 \sigma} G^{r} \Gamma_{1} G^{a}\right)$. The corresponding spin Hall conductance fluctuation is defined as $\operatorname{rms} G_{s H}=$ $\sqrt{\left\langle G_{s H}^{2}\right\rangle-\left\langle G_{s H}\right\rangle^{2}}$, where $\langle\cdots\rangle$ denotes averaging over an ensemble of samples with different disorder configurations of same strength $W$. Numerical calculations were performed on $L \times L$ square samples of size $L=40$ up to 100 . We measure the electron energy $E$, disorder strength $W$, and Rashba spin-orbit coupling $t_{S O}$ etc. in unit of the hopping energy $t$ (same for the following section). In the presence of spin-orbit coupling, spin is no longer a good quantum number, ${ }^{23,26}$ so in order to make the definition of the spin current meaningful, we will set the spin-orbit coupling to be zero in the detecting probes, i.e. probes 2 and 4 .

\subsubsection{Quantum spin-Hall effect models}

Kane and Mele ${ }^{7,8}$ proposed that introducing the local time-reversal symmetry breaking on the two sublattices of graphene via intrinsic spin-orbit coupling could open an energy gap and give rise to the quantum spin-Hall effect. Distinct from 
the conventional spin-Hall effect, the quantized spin-Hall effect is a time-reversal symmetry protected insulator exhibiting helical edge states with opposite spins propagating in opposite directions along the same boundary, which is schematically shown in Fig. 5(a) in a four-probe Hall bar setup. This model immediately attracted numerous attention thereafter, and later first principles calculations showed that the intrinsic spin-orbit coupling is extremely small — about $10^{-3} \mathrm{meV} \cdot{ }^{27,28}$ Though the intrinsic spin-orbit coupling induced quantum spin-Hall effect in graphene model is unrealistic nowadays with experimental technique, it nonetheless initiated the research field of three-dimensional topological insulator, which has become a tremendous focus in the condensed matter community. ${ }^{10,11}$

Later on, another group of material, HgTe quantum wells, ${ }^{9}$ was also reported to show quantum spin-Hall effect. Fortunately, the HgTe model has been experimentally observed to exhibit quantized longitudinal resistance, which is directly related to the quantized spin-Hall effect. ${ }^{29}$ In the quantum spin-Hall effect and the three-dimensional topological insulators, time-reversal symmetry is required to be preserved. In fact, this is not mandatory in our investigation of the universality from the quantized spin-Hall conductance. Thus, a third model of graphene in the presence of magnetic field ${ }^{30,31}$ will also be discussed. From the viewpoint of the symmetry, model I (graphene with intrinsic spin-orbit coupling) belongs to CSE; model II (graphene due to strong magnetic field and Zeeman effect) belongs to CUE; while the third model (HgTe material) belongs to CSE. The detailed tight-binding Hamiltonian of these models will be introduced in Sec. 3 .

\subsubsection{Random matrix theory}

Random matrix theory is a powerful tool of mathematical physics developed since the 1960s, which deals with statistical properties of matrices with randomly distributed elements. ${ }^{32}$ The physical properties are computed from the correlation functions of eigenvalues and eigenvectors. Energy levels of heavy nuclei measured in nuclear reactions and small metal particles for microwave absorption were understood in terms of statistics of random matrix. Then random matrix theory has been applied to study the statistics of quantum transport properties of mesoscopic systems, such as chaotic cavities and disordered wire. ${ }^{17}$ The scattering matrix $S$ of open system which determines the conductance through Landauer formula $G=\left(2 e^{2} / h\right) \operatorname{Tr}\left[t t^{\dagger}\right]$ is chosen from a simple statistical ensemble with only symmetry constraints applied. This is for scattering involving leads with $N$ channels and width $L, S=\left(\begin{array}{cc}r & t^{\prime} \\ t & r^{\prime}\end{array}\right)$, where $r, t$ are $N \times N$ reflection and transmission matrices. One can replace the scattering matrix $S$ by a random matrix taken from the proper Dyson's circular ensemble. Then the generalized transmission probability can be written as a trace over $S$. The average, variance and covariance of the transmission over the circular ensemble can then be derived from the method of Ref. 33. Random matrix theory relates the universality of transport properties to the universality of 
correlation function of transmission eigenvalues. It is general and applies to a class of transport problems, and provides a unified description from metallic to localized regimes.

\section{Universal Spin-Hall Conductance Fluctuation for Conventional Spin-Hall Effect}

In the presence of spin-orbit coupling, chemical potentials of the spin-up and spin-down channels become different at two edges of a mesoscopic sample with finite width. Based on the above mentioned calculation methods, a universal value $\sim 0.18 e / 4 \pi$ for the spin Hall conductance fluctuation in disordered samples was predicted in 2006. ${ }^{14}$ This value is independent of the system details, such as Fermi energy, disorder strength, spin-orbit coupling strength, and system size.

Figure 2 plots the spin-Hall conductance fluctuation as a function of the Fermi energy at fixed $t_{S O}=0.3$ (Rashba spin-orbit interaction only) and the sample size $L=40$ for several disorder strengths $W=1,2,3$, and the corresponding averaged

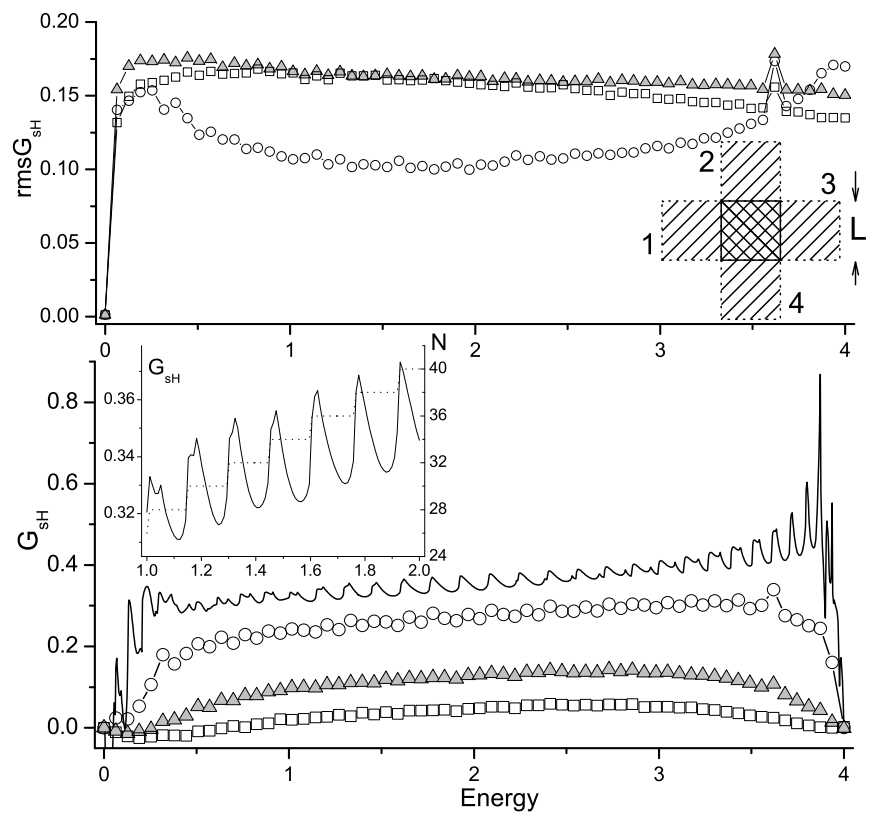

Fig. 2. Spin-Hall conductance fluctuation versus Fermi energy for disordered samples. Triangles, squares, and circles are for $W=1,2,3$, respectively. Upper inset: schematic plot of the four-terminal mesoscopic sample where Rashba spin-orbit coupling exists in the center scattering region and the leads 1 and 3 . The width of the square sample is $L=40$. A small voltage bias is across leads 1 and 3. Spin-Hall conductance is measured through leads 2 and 4 . Lower panel: the ensemble averaged spin-Hall conductance $G_{s H}$ versus Fermi energy for spin-orbit coupling strength $t_{S O}=0.3$. The solid line is for pure sample with $W=0$ and other symbols are the same as the upper panel. In all the figures the spin conductance and its fluctuation are measured in units of $e / 4 \pi$. 


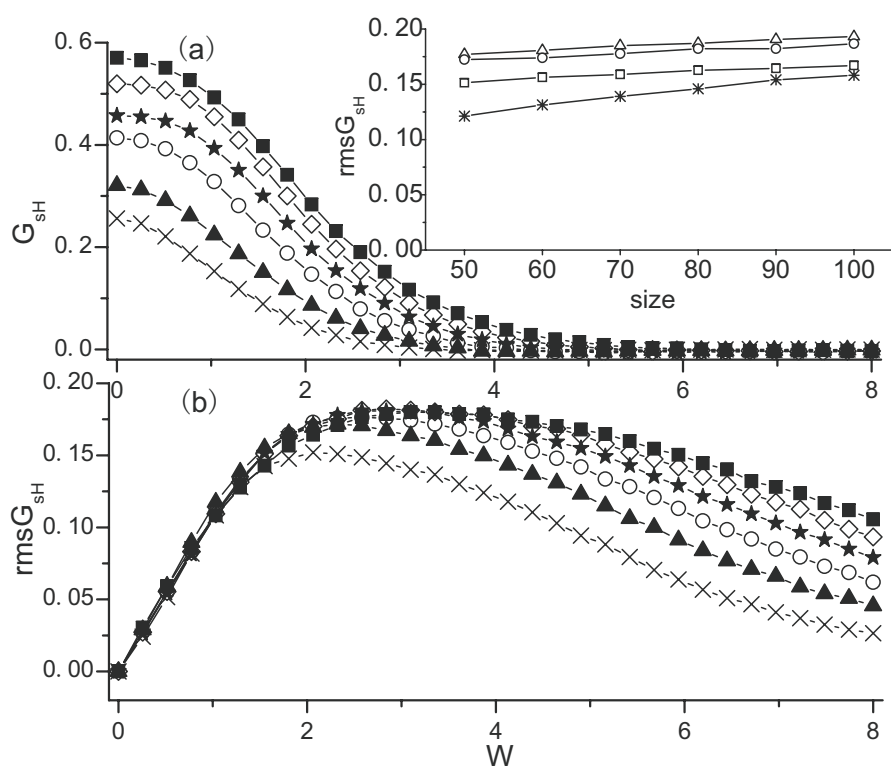

Fig. 3. (a) Ensemble averaged spin-Hall conductance $G_{s H}$ versus disorder strength $W$ for $t_{S O}=$ 0.2 (cross), 0.3 (solid triangle), 0.4 (open circle), 0.5 (star), 0.6 (rumbus), and 0.7 (solid square). The average is taken over 20,000 samples with $L=40$. Inset: size dependence of the spin-Hall conductance fluctuation with $t_{S O}=0.3$. Different symbols are for $W=1$ (stars), 2 (rectangles), 3 (circles), and 4 (triangles). The ensemble average is over 20,000 samples for different size $L$. (b) The corresponding ensemble averaged spin-Hall conductance fluctuation versus $W$, and the symbols are for the same $t_{S O}$ values as in panel (a).

spin-Hall conductance in the lower panel from 10,000 disordered samples. For pure samples without disorder, as the Fermi energy increases, the number of subbands increases (see the inset of the lower panel). As a result, the spin-Hall conductance $G_{s H}$ shows small oscillations. When disorder is increased from zero, $G_{s H}$ decreases as expected, and eventually the small oscillation due to the subbands vanishes. Most importantly, Fig. 2 shows substantial sample-to-sample fluctuations of $G_{s H}$, measured by $\operatorname{rms} G_{s H}$, of the order $\delta e / 4 \pi$, where $\delta$ is a number between 0.1 and 0.2. Remarkably, such an amplitude of fluctuation is comparable to the spin-Hall conductance itself.

Figure 3 plots the averaged spin-Hall conductance $G_{s H}$ and corresponding fluctuations $\operatorname{rms}\left(G_{s H}\right)$ as a function of disorder strength $W$ at fixed Fermi energy $E=1$ for a number of different spin-orbit couplings from $t_{S O}=0.2$ up to 0.7 . The spin-Hall conductance decreases smoothly as the disorder strength is increased: the transport characteristics change from quasiballistic at small $W$ to the diffusive regime at larger $W=[1,5]$. Finally it goes into the insulating regime for even larger $W$ where $G_{s H}$ vanishes. The fluctuations develop a plateau structure so that $\operatorname{rms}\left(G_{s H}\right)$ becomes independent of the disorder parameter $W$ for each given $t_{S O}$. In this sense, the fluctuation $\operatorname{rms}\left(G_{s H}\right)$ becomes "universal" and the spin-Hall 


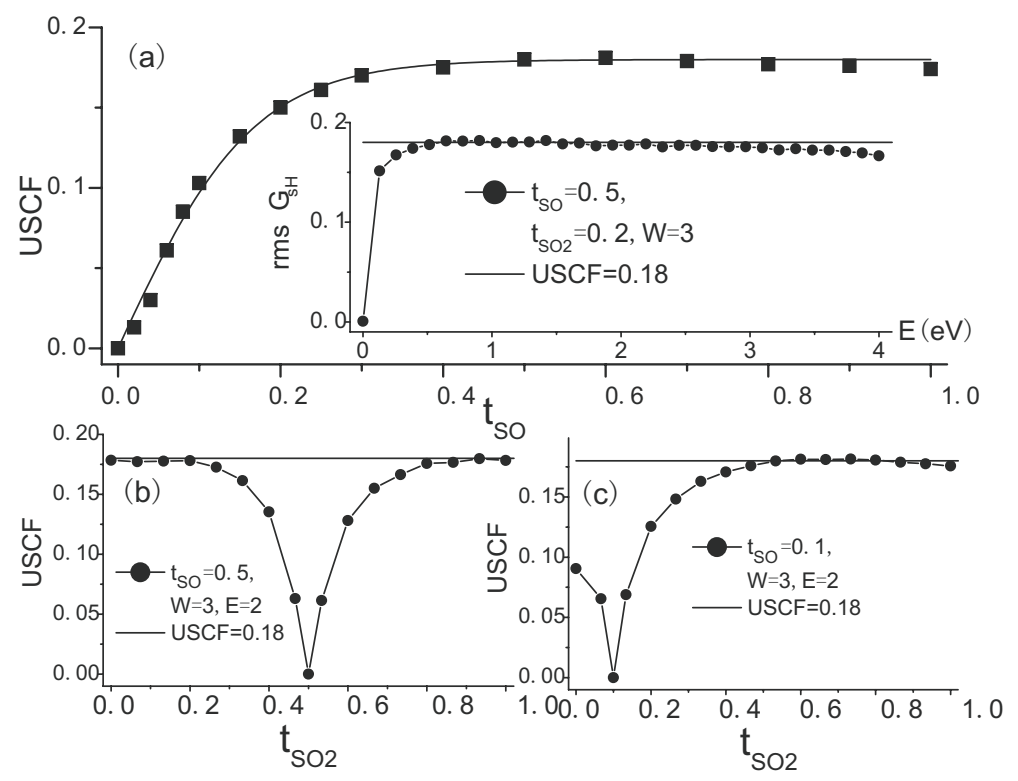

Fig. 4. (a) USCF values versus Rashba spin-orbit coupling $t_{S O}$ at $E=1$. Inset: $\operatorname{rms}\left(G_{S H}\right)$ versus Fermi energy for $W=3$ in the presence of both Rashba and Dresselhuss SO couplings, $t_{S O}=0.5$ and $t_{S O 2}=0.2$. (b) and (c) $\operatorname{rms}\left(G_{s H}\right)$ versus Desselhaus $\mathrm{SO}$ coupling $t_{\mathrm{SO} 2}$ at $E=2, W=3$, and $t_{S O}=0.5 / t_{S O}=0.1$. In the cases of inset of (a), (b) and (c), 40,000 samples are collected.

transport enters the regime with USCF. It is notable that the larger the $t_{S O}$, the wider the fluctuation plateau which characterizes the diffusive regime.

An examination at the dependence of spin-Hall conductance fluctuation $\operatorname{rms}\left(G_{s H}\right)$ on system size $L$ is shown in the inset of Fig. 3 for $t_{S O}=0.3$. With weak disorder $W=1$ (stars) the fluctuation increases with sample size indicating that spin-Hall conductance is not yet in the USCF regime because transport is quasiballistic. In the diffusive regime, $W=2,3,4$, the fluctuations saturate at $\delta e / 4 \pi$ where $\delta \simeq 0.2$. The independence of system size by the fluctuation $\operatorname{rms}\left(G_{s H}\right)$ provides strong evidence of USCF. Namely, as long as transport is in the diffusive regime, the fluctuation of the spin-Hall conductance is dominated by quantum interference giving rise to a universal amplitude. Since USCF should disappear when the spin-orbit coupling $t_{S O}$ and thus $G_{s H}$ are zero, a collection of the USCF for different $t_{S O}$ is shown in Fig. 4. Interestingly, the USCF can be well fitted (solid line) by a function $\operatorname{rms}\left(G_{s H}\right)=g \tanh \left(t_{S O} / 0.17\right)$, where $g=0.18 e / 4 \pi$. A similar case happens when we include Dresselhaus spin-orbit coupling by adding a term $\beta_{S O}\left(\sigma_{x} k_{x}-\sigma_{y} k_{y}\right)$ in the Hamiltonian. It can be proven that the spin-Hall current along the $z$ direction persists only when the Rashba and Dresselhaus strengths do not equal, ${ }^{34}$ because these two terms have different symmetries: the Rashba coupling arises from the structure inversion asymmetry with $\mathrm{SU}(2)$ symmetry, while the Dresselhaus coupling arises from the bulk inversion asymmetry with SU(1,1) symmetry. ${ }^{35}$ From Fig. 4, we see that vanishing spin current causes a dip in USCF 
curves whenever $\alpha_{S O}=\beta_{S O}$. Finally, the inset of Fig. 4 shows that the USCF is independent of Fermi energy even when both SO interactions are present.

It was then quantitatively confirmed and beautifully understood by RMT the analysis results. From Eq. (11) of Ref. 15,

$$
\operatorname{var}\left[J_{i}\right]=\frac{4 N_{i} N_{l} N_{r}\left(N_{T}-1\right)}{N_{T}\left(2 N_{T}-1\right)\left(2 N_{T}-3\right)\left(N_{l}+N_{r}\right)}
$$

where $N_{T}=\sum_{i=1,4} N_{i}$ is the total channels of all electrodes. For our fully symmetric configuration, $N_{i}=N$, the spin current fluctuates universally for large $N$, with $\operatorname{rms}\left(I_{s H}\right)=\left(e^{2} V / h\right) / \sqrt{32}$. This can be translated into the universal fluctuations of the transverse spin Hall conductance with

$$
\operatorname{rms}\left(G_{s H}\right)=e / 4 \pi \sqrt{32}=0.18(e / 4 \pi) .
$$

Furthermore, the mesoscopic fluctuation problem of spin current has also been investigated by using another different approach called quantum circuit theory. ${ }^{36}$ Very recently, some theoretical research showed that zigzag edge graphene nanoribbons could have zero spin conductance but nonzero spin Hall conductance. ${ }^{37,38}$ And graphene nanoribbons with rough edges were found to exhibit mesoscopic spin conductance fluctuations with a universal value of $\operatorname{rms}\left(G_{s}\right)=$ $0.4 e / 4 \pi$ in the diffusive regime. ${ }^{38}$ In a three-terminal set-up, random matrix theory has also predicted that the spin conductance of chaotic ballistic cavity fluctuates universally $\operatorname{rms}\left(G_{s}\right)=0.27 e / 4 \pi$ about zero mesoscopic average. ${ }^{39}$

\section{Universal Spin-Hall Conductance Fluctuation for Quantized Spin-Hall Effect}

\subsection{Graphene model}

Graphene is a recently discovered two-dimensional one-atom-thick material consisting of a honeycomb lattice of carbon atoms. It exhibits excellent mechanical properties under stress and electrical conduction properties under electrostatic bias. ${ }^{40}$ What makes graphene so unique is that it has a linear Dirac-type dispersion at two inequivalent points $K$ and $K^{\prime}$ at Brillouin zone corners. In Sec. 3.1.1, we investigate universal spin-Hall conductance fluctuation of the quantized spin-Hall conductance of graphene model with intrinsic spin-orbit interactions, where the spin-rotation symmetry is broken and the system possesses CSE. In Sec. 3.1.2, we study the other graphene model under strong magnetic field and Zeeman effects, where the time-reversal symmetry is broken and the system belongs to CUE.

\subsubsection{Quantized spin-Hall effect due to intrinsic spin-orbit coupling}

In graphene, there are two kinds of spin-orbit coupling: intrinsic and Rashba spin-orbit couplings. ${ }^{7}$ The intrinsic one originates from the in-plane structural asymmetry, while the Rashba one arises from the mirror symmetry breaking 

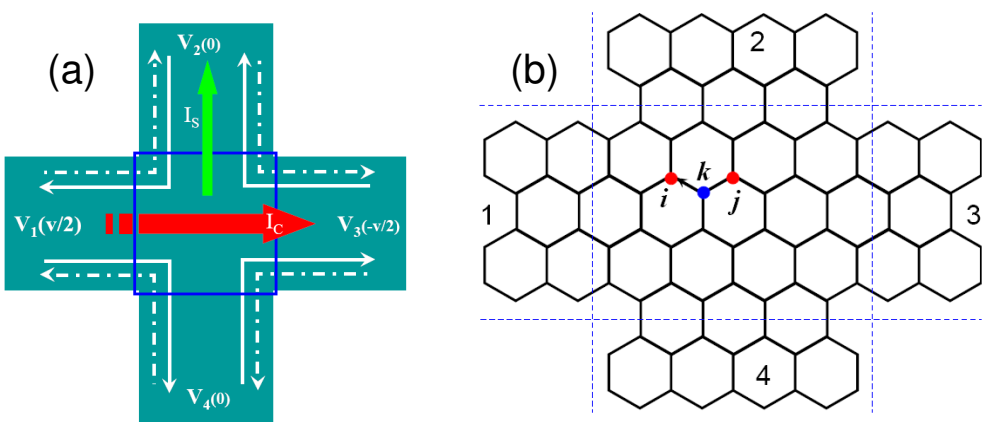

Fig. 5. (Color online) (a) Edge state propagation in the quantum spin-Hall setup. Solid/dashed lines stand for spin-up/spin-down states. The voltages are set to be $V_{i}=(V / 2,0,-V / 2,0)$, with $i=1,2,3,4$. (b) Schematic plot of the four-terminal mesoscopic graphene sample where the intrinsic spin-orbit coupling exists in the whole regimes, while the Rashba spin-orbit coupling only appears in the central scattering regime and leads 1 and 3 . Leads 2 and 4 are used to measure the spin-current.

of the graphene plane (i.e. by the external electric field or by the interaction with a substrate, as discussed above). The first-principles calculations show that the Rashba spin-orbit coupling is larger than the intrinsic one. ${ }^{27,28}$ Recent angle-resolved photoemission experiments suggest that graphene's Rashba type spin-orbit coupling strength can be enhanced to a sizeable $10 \mathrm{meV}$ by surface doping with impurity atoms. ${ }^{41-43}$ This has led to a resurgence of excitement and interest in Rashba spin-orbit coupling effects in graphene. ${ }^{44}$ Although the realization of intrinsic spin-orbit coupling induced quantized spin-Hall effect is impossible under experimental conditions so far, it is still a good toy model to numerically study the transport properties of the quantized spin-Hall effect. And more importantly, this model is the only system (compared to other topological insulators) in which the spin is a good quantum number.

Again in the tight-binding representation, the Hamiltonian for the single layer graphene in the presence of intrinsic and Rashba spin-orbit couplings can be written $\mathrm{as}^{8,45}$ :

$$
\begin{aligned}
H= & -t \sum_{\langle i j\rangle} c_{i}^{\dagger} c_{j}+\frac{2 i}{\sqrt{3}} V_{S O} \sum_{\langle\langle i j\rangle\rangle} c_{i}^{\dagger} \sigma \cdot\left(\mathbf{d}_{k j} \times \mathbf{d}_{i k}\right) c_{j} \\
& +i V_{r} \sum_{\langle i j\rangle} c_{i}^{\dagger} \hat{\mathbf{e}}_{z} \cdot\left(\sigma \times \mathbf{d}_{i j}\right) c_{j}+\sum_{i} \epsilon_{i} c_{i}^{\dagger} c_{i} .
\end{aligned}
$$

The first term is the nearest hopping term, the second one is the intrinsic spin-orbit interaction that involves the next nearest sites. As shown in Fig. 5, $i$ and $j$ are two next nearest neighbors, $k$ is the common nearest neighbor of $i$ and $j$, and $\mathbf{d}_{i k}$ describes a vector pointing from $k$ to $i$. The third term is the Rashba spin-orbit coupling, and the last term on-site energy $\epsilon_{i}$ is a random potential uniformly distributed in $[-W / 2, W / 2]$. Because intrinsic spin-orbit coupling is a 

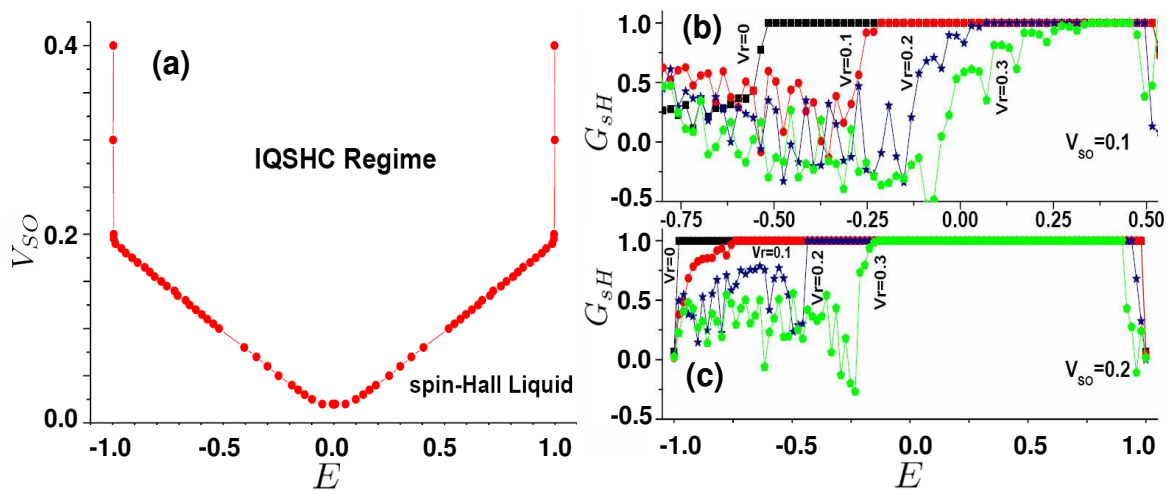

Fig. 6. (Color online) (a) Phase-diagram of the quantized spin-Hall conductance regime (IQSHC) in the plane $\left(E, V_{S O}\right)$. (b) and (c) Spin-Hall conductance $\left\langle G_{s H}\right\rangle$ versus Fermi energy $E$ at fixed intrinsic spin-orbit coupling $V_{S O}=0.1,0.2$ for different $V_{R}=0,0.1,0.2,0.3$, respectively.

good quantum number, but the Rashba spin-orbit coupling is not, so the intrinsic spin-orbit coupling exists in the whole regime while the Rashba spin-orbit coupling only exists in the scattering regime and the leads 1 and 3. Leads 2 and 4 are used to measure the spin-current in our measuring setup shown in Fig. 5.

Figure 6(a) shows the phase diagram of the quantized spin-Hall conductance in $\left(E, V_{S O}\right)$ space in the absence of Rashba spin-orbit coupling. One observes that when the intrinsic spin-orbit coupling $V_{S O}<0.2$, the phase boundary is nearly linear; when $V_{S O}>0.2$, the phase boundary is a constant at $E= \pm 1.0$. In the panels (b) and (c), the quantized spin-Hall conductance is gradually destroyed by the increasing Rashba spin-orbit coupling. One further finds that the quantized spin-Hall conductance more easily destroyed in the hole-carrier regime. In the following, we shall focus on the numerical investigation on the "destroyed" quantum spin-Hall effect by disorders in the diffusive regime.

When the disorder strength $W$ increases, the graphene system is at first in the ballistic regime for the weak disorders: $\left\langle G_{s H}\right\rangle=e / 4 \pi$ and $\operatorname{rms}\left(G_{s H}\right)=0$; for the intermediate disorders, the quantization of $\left\langle G_{s H}\right\rangle$ breaks down due to a direct propagation from the leads 1 to 3 , and $\operatorname{rms} G_{s H}$ begins to increase; for the even larger disorder $W$, it goes into the insulating regime, where both $\left\langle G_{s H}\right\rangle$ and $\operatorname{rms}\left(G_{s H}\right)$ vanish. For example, Fig. 7 plots the averaged spin-Hall conductance and its fluctuation at fixed Fermi energy $E=0.5$ for different intrinsic spin-orbit couplings $V_{S O}=0.1,0.3,0.7$ and 0.9 , respectively. One observes that the quantized spin-Hall conductance is very robust against disorders and can survive up to $W=3$ for $V_{S O}=0.3,0.7$ and 0.9 , with the corresponding spin-Hall conductance fluctuation being exactly zero. When the disorder strength is further increased, the quantized spin-Hall conductance is gradually decreased, while the fluctuations $\operatorname{rms}\left(G_{s H}\right)$ are gradually increased. In panel (b), one can find that all the four curves reach the same maximal value at different disorder ranges $\left(\operatorname{rms}\left(G_{s H}\right)=0.285\right.$ in unit of 


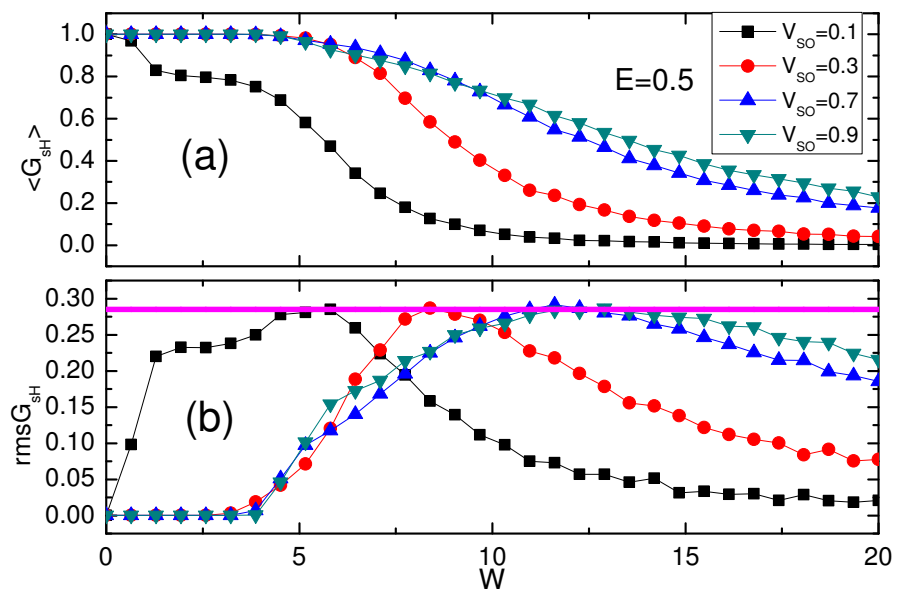

Fig. 7. (Color online) (a) The averaged spin-Hall conductance $\left\langle G_{s H}\right\rangle$ and (b) its fluctuation $\operatorname{rms}\left(G_{s H}\right)$ versus disorder strength $W$ at the fixed Fermi energy $E=0.5$ for different intrinsic spin-orbit couplings $V_{S O}=0.1,0.3,0.7,0.9$, respectively. 10,000 samples are collected for each curve.

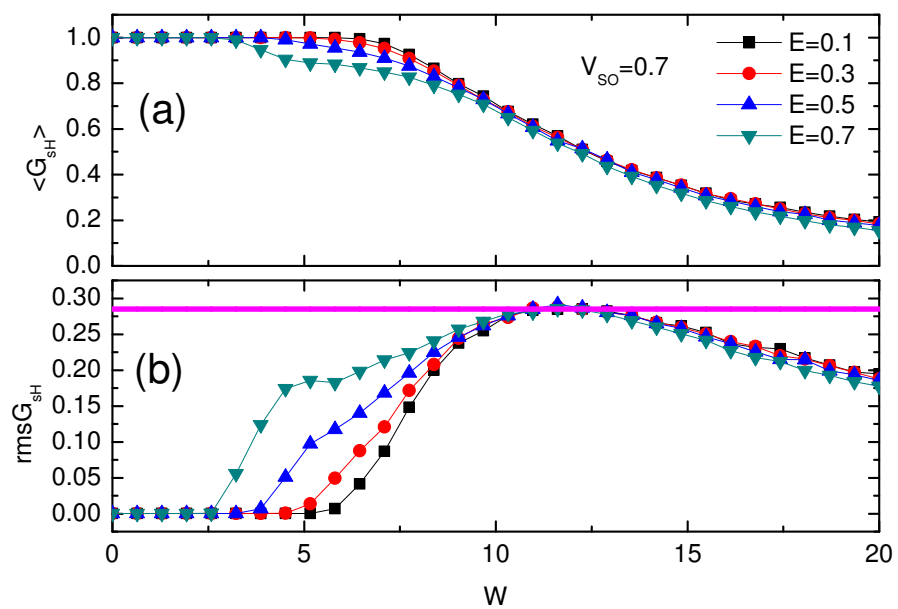

Fig. 8. (Color online) (a) The averaged spin-Hall conductance $\left\langle G_{s H}\right\rangle$ and (b) its fluctuation $\operatorname{rms}\left(G_{s H}\right)$ versus disorder strength $W$ at the fixed intrinsic spin-orbit coupling $V_{S O}=0.7$ for different Fermi energies $E=0.1,0.3,0.5,0.7$. 10,000 samples are collected for each curve.

$e / 4 \pi)$. Although the plateau range of $W$ depends on specific values of $E$ or $V_{S O}$, our results show that it always resides in the diffusive regime.

In Fig. 8, we show the spin-Hall conductance and the its fluctuation as a function of disorder strength at the fixed intrinsic spin-orbit coupling for different Fermi energies $E=0.1,0.3,0.5,0.7$. In panel (a), one can find that the Fermi energy near the gap center is more robust than other Fermi energies for the fixed intrinsic spin-orbit coupling. Similar to Fig. 7(b), we observe that the four curves for different 


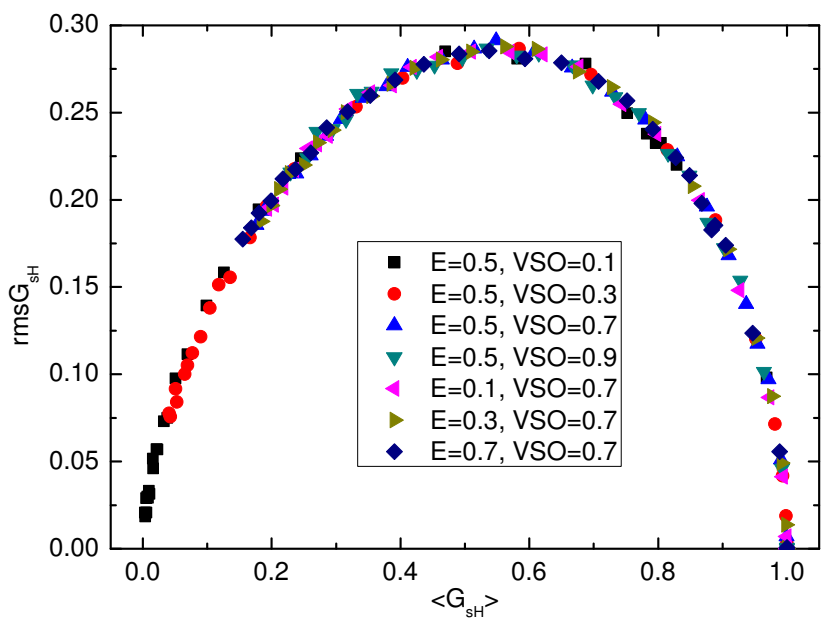

Fig. 9. (Color online) The spin-Hall conductance fluctuation $\left(\operatorname{rms}\left(G_{s H}\right)\right)$ versus the averaged $\left\langle G_{s H}\right\rangle$ for various Fermi energies $E$ and intrinsic spin-orbit coupling $V_{S O}$.

Fermi energies share the same plateau $\operatorname{rms}\left(G_{s H}\right)=0.285 e / 4 \pi$ at the same disorder range. From Figs. 7 and 8 , we find that $\operatorname{rms}\left(G_{s H}\right)=0.285$ is always true if there is a plateau, i.e. if the diffusive transport regime is established. We therefore identify $\operatorname{rms}\left(G_{s H}\right)=0.285$ as a "universal" value, which is independent of the Fermi energy, and the intrinsic spin-orbit coupling strength.

To confirm this universal characteristic of spin-Hall conductance fluctuation, we can also directly plot the relation between the averaged spin-Hall conductance $\left\langle G_{s H}\right\rangle$ and the fluctuation $\operatorname{rms}\left(G_{s H}\right)$ as in the study of the universal conductance fluctuation. ${ }^{18,46}$ Figure 9 illustrates that all the data in Figs. 7 and 8 collapse into a single smooth curve, where $\operatorname{rms}\left(G_{s H}\right)$ is correlated with $\left\langle G_{s H}\right\rangle$ in the ballistic, diffusive, and insulating spin-transport regimes. We now conclude that the quantized spin-Hall effect in the graphene model due to the intrinsic spin-orbit coupling has its own USCF and is quite different from that in the conventional spin-Hall effect. In order to make a connection between the two universality classes, it is necessary to investigate the behavior of the spin-Hall liquid as shown in Fig. 6(a). In Fig. 10, we show the averaged conductance and fluctuation as functions of disorder strength for some large Fermi energies $(E=1.1,1.5,2.0)$ at fixed intrinsic spin-orbit coupling $V_{S O}=0.7$. From these parameters, the spin-Hall conductance is no longer quantized in the absence of disorder. The spin-Hall conductance fluctuations reach the plateau $\sim 0.18 e / 4 \pi$, which is exactly the same as USCF found in the conventional spin-Hall effect of Sec. 2.

\subsubsection{Quantized spin-Hall effect under magnetic field and Zeeman effect}

In Sec. 3.1.1, we have seen that the quantized spin-Hall conductance fluctuation is independent on the system details. In order to examine the model dependence of 


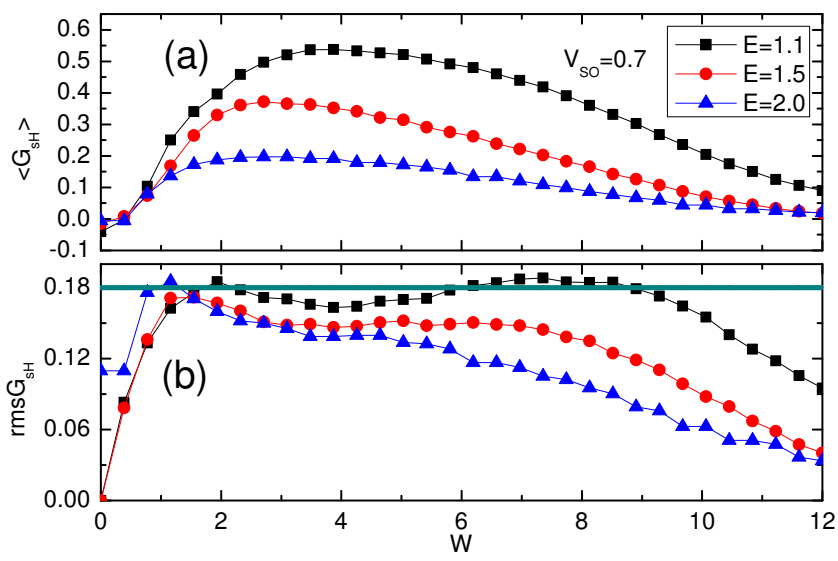

Fig. 10. (Color online) (a) The averaged spin-Hall conductance $\left\langle G_{s H}\right\rangle$ and (b) its fluctuation $\operatorname{rms}\left(G_{s H}\right)$ versus disorder strength $W$ at the fixed intrinsic spin-orbit coupling $V_{S O}=0.7$ for some large Fermi energies $E=1.1,1.5,2.0$, where the spin-Hall conductance is not quantized in the absence of disorders. 10,000 samples are collected for each curve.

the USCF for the quantized spin-Hall effect, the investigation on a second model is desirable. In this section, we consider a graphene model in the presence of strong magnetic field and Zeeman effect. Unlike the above time-reversal invariant quantum spin-Hall state, this time-reversal asymmetric model (CUE) shows the quantized spin-Hall conductance due to edge states in the quantum Hall regime. Because of the Zeeman splitting and graphene's linear energy spectrum, both electron-like and hole-like edge states exist near the Fermi level forming the counter-circulating edge states that have been confirmed experimentally. ${ }^{30,31}$

As shown in Fig. 11, when a voltage bias is applied across probes 1 and 3, a transverse flow of dissipationless spin-current can be generated, i.e. only spin-down current propagates from lead 1 to lead 2, and spin-up current flows from lead 3 to lead 2. Such a quantized spin-Hall conductance breaks down with the sample-to-sample fluctuations, as the disorder strength $W$ increases. Figure 12 plots the averaged spin-Hall conductance and corresponding fluctuations as functions of disorder strength $W$ for different parameters. From panels (c) and (d), one can find that for a given $E$ or $\phi, \operatorname{rms}\left(G_{s H}\right)$ develops a plateau region, e.g. in the range $W=(3,7)$ in panel (c), exhibiting the same value $0.285 e / 4 \pi$. Therefore, results of two different models strongly suggest that the quantized spin-Hall effect belongs to a new universality class with $\mathrm{USCF}_{2}=0.285 e / 4 \pi$ in contrast to $\mathrm{USCF}=0.18 e / 4 \pi$ for the conventional spin-Hall effect.

\subsection{HgTe - A square lattice model}

The above two models are both related to honeycomb lattice with the special Dirac dispersion of graphene. In this section, we address a third model — square lattice 

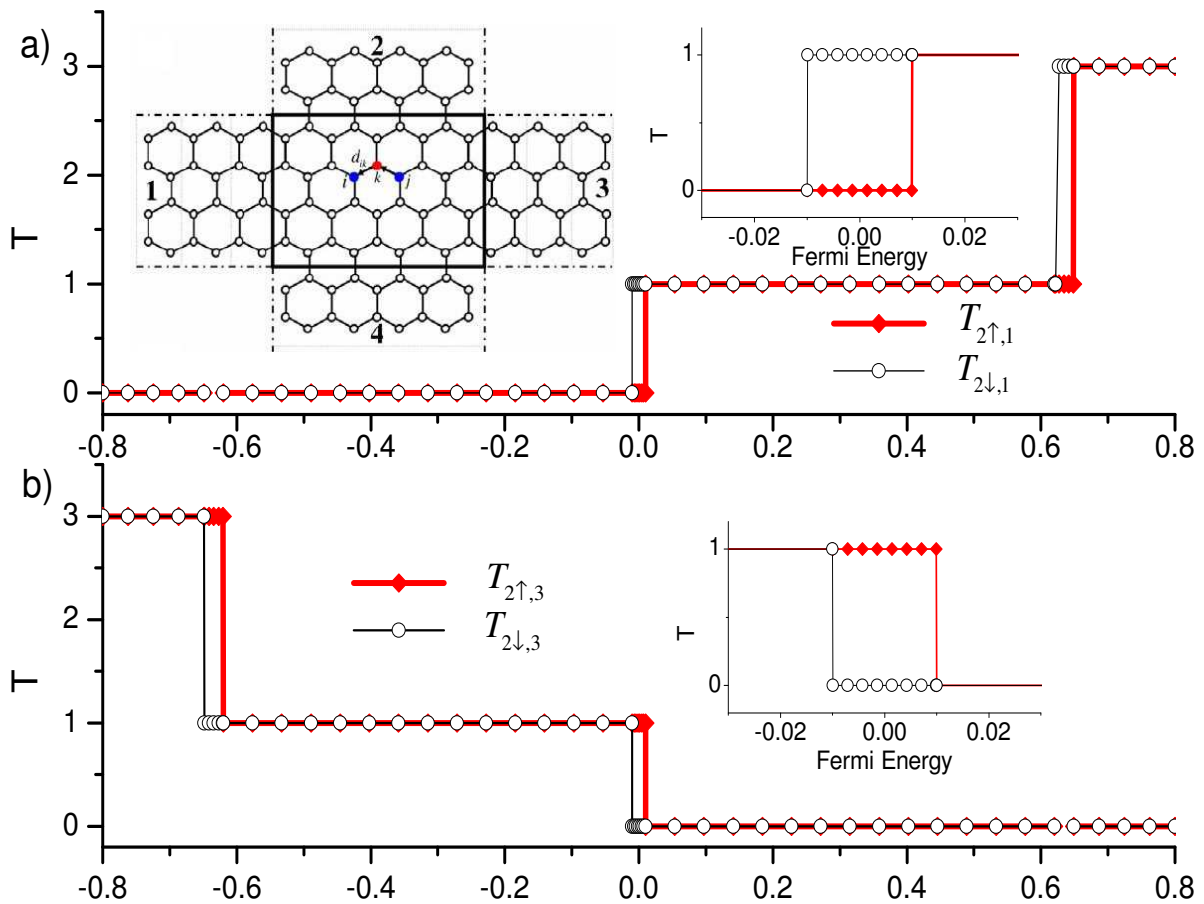

Fermi Energy

Fig. 11. (Color online) (a) Transmission coefficients $T_{2 \uparrow / \downarrow, 1}$ and (b) $T_{2 \uparrow / \downarrow, 3}$ versus Fermi energy at fixed magnetic flux $\phi=0.08$. Insets are the zooming in near the Fermi energy $E=0$.

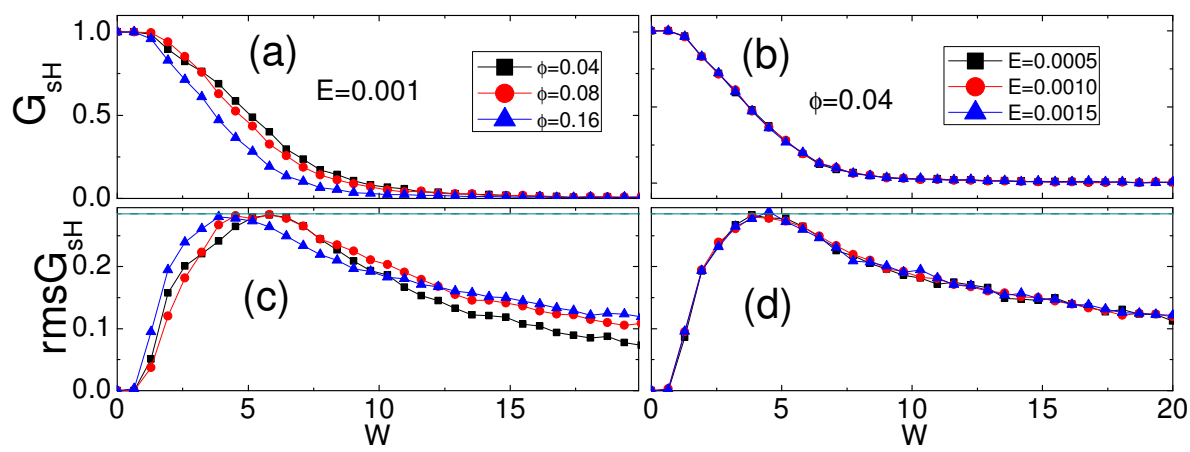

Fig. 12. (Color online) (a) and (b) Averaged spin-Hall conductance and fluctuations at fixed Fermi energy $E=0.001$ for different magnetic flux $\phi=0.04,0.08,0.16$. (c) and (d) Averaged spin-Hall conductance and fluctuations at fixed magnetic flux $\phi=0.04$ for different Fermi energies $E=0.0005,0.0010,0.0015$.

HgTe model (CSE), and the effective Hamiltonian can be written as ${ }^{9,29,47}$ :

$$
H=\left(\begin{array}{cc}
H(k) & 0 \\
0 & H^{*}(-k)
\end{array}\right)
$$




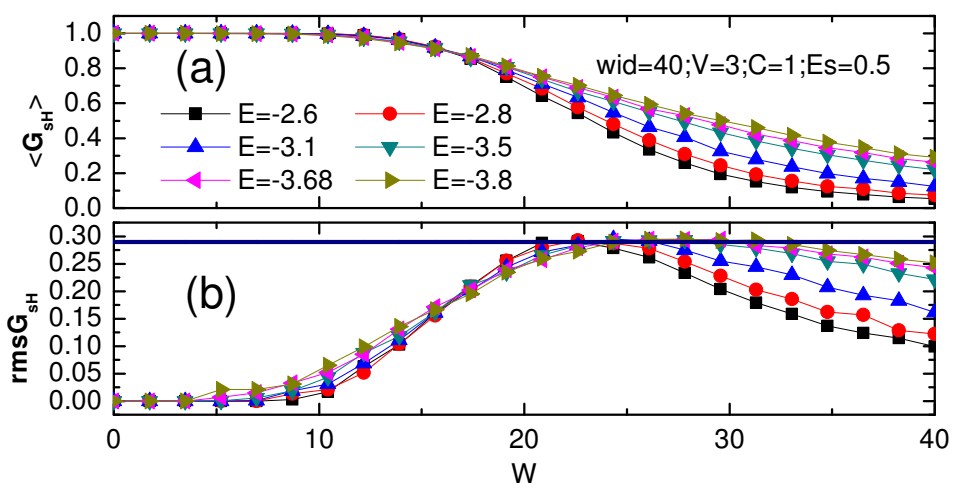

Fig. 13. (Color online) (a) and (b) Averaged spin-Hall conductance and fluctuations of the $\mathrm{HgTe}$ model as a function of disorder strength for different Fermi energies $E=-2.6$, $-2.8,-3.1,-3.5,-3.68,-3.8$ at fixed $V=3, c=1$, and $e_{s}=0.5$. The system size is $L=40$.

where each of the diagonal block exhibits quantum anomalous Hall effect, and they are chirally opposite. Thus the chirality of each block acts as the spin degree of freedom. In the tight-binding approximation, the Hamiltonian can be written as:

$$
\begin{aligned}
H= & -t \sum_{\langle i j\rangle}\left(c_{i}^{\dagger} c_{j}+\text { h.c. }\right)+\frac{i V}{2} \sum_{i}\left(c_{i}^{\dagger} \sigma_{y} c_{i+\hat{x}}-c_{i}^{\dagger} \sigma_{x} c_{i+\hat{y}}+\text { h.c. }\right) \\
& -\frac{c V}{2} \sum_{\langle i j\rangle}\left(c_{i}^{\dagger} \sigma_{z} c_{j}+\text { h.c. }\right)+\left(2-e_{s}\right) V c \sum_{i} c_{i}^{\dagger} \sigma_{z} c_{i} .
\end{aligned}
$$

As shown in Fig. 13, the spin-Hall conductance for the quantized spin-Hall conductance in the diffusive regime reach the same plateau regime $\operatorname{rms}\left(G_{s H}\right)=$ $0.285 e / 4 \pi$ again. So briefly we have confirmed $\mathrm{USCF}_{2}$ for the quantized spin-Hall effect using three different models: two graphene modes with CSE and CUE symmetries, and a third square lattice model with CSE symmetry. All of them share the same plateau peak value in the diffusive regimes independent on all system/model details.

\section{Conclusion and Perspectives}

Since universal conductance fluctuation has long been recognized for its importance, we believe that the universal spin Hall conductance fluctuation (USCF) will be a hallmark in the modern spintronics field. In brief, this review illustrates a prominent feature in the electron transport where spin is dominant. The USCF $\sim 0.18 e / 4 \pi$ has been obtained from numerical method and verified by the analytic theoretical approach. The presented universal quantized spin Hall conductance $0.285 e / 4 \pi$ is also expected to be confirmed by other theoretical and experimental measurement work in the future. Some closely relevant spin-resolved transport study based on graphene nanoribbons has recently added more interest to the universality discussed here. 
To summarize, we have reviewed the universal spin-Hall conductance fluctuations for the conventional and quantized spin-Hall effect. We conclude that they belong to two different universality classes. In the current study, we have mainly focused on the two-dimensional systems. During the time of writing of this review, the study of the three-dimensional topological insulators has become a demanding task. Furthermore, previously we have only considered the spin-independent disorder. On the other hand, the magnetic and spin-flip types of disorders may bring new avenues to the investigation of the universality classes of the spin-related conductance.

\section{Acknowledgments}

This work was supported by RGC grant (HKU7054/09P) from the government of Special Administration Region (SAR) of Hong Kong and LuXin Energy Group. The Computer Center of the University of Hong Kong are gratefully acknowledged for computing assistance.

\section{References}

1. I. Zutic, J. Fabian and S. Das Sarma, Rev. Mod. Phys. 76 (2004) 323.

2. J. E. Hirsch, Phys. Rev. Lett. 83 (1999) 1834.

3. J. Sinova, D. Culcer, Q. Niu, N. A. Sinitsyn, T. Jungwirth and A. H. MacDonald, Phys. Rev. Lett. 92 (2004) 126603.

4. Y. K. Kato, R. C. Myers, A. C. Gossard and D. D. Awschalom, Science 306 (2004) 1910.

5. J. Wunderlich, B. Kaestner, J. Sinova and T. Jungwirth, Phys. Rev. Lett. 94 (2005) 047204 .

6. M. I. Dyakonov and V. I. Perel, Phys. Lett. A 35 (1971) 459.

7. C. L. Kane and E. J. Mele, Phys. Rev. Lett. 95 (2005) 226801.

8. C. L. Kane and E. J. Mele, Phys. Rev. Lett. 95 (2005) 146802.

9. B. A. Bernevig, T. L. Hughes, Science 314 (2006) 1757.

10. L. Fu et al., Phys. Rev. Lett. 98 (2007) 106803; J. E. Moore and L. Balents, Phys. Rev. B 75 (2007) 121306(R); X.-L. Qi et al., ibid. 78 (2008) 195424; R. Roy, ibid. 79 (2009) 195321; H. Zhang et al., Nat. Phys. 5 (2009) 438; D. Hsieh et al., Nature 452 (2008) 970; Y. Xia et al., Nat. Phys. 5 (2009) 398; Y. L. Chen et al., Science 325 (2009) 178.

11. M. Z. Hasan and C. L. Kane, Rev. Mod. Phys. 82 (2010) 3045; X.-L Qi and S.-C. Zhang, Phys. Today 63 (2010) 33; X.-L. Qi and S.-C. Zhang arXiv:1001.1602; J. E. Moore, Nature 464 (2010) 194; M. Z. Hasan and J. E. Moore, arXiv:1011.5462.

12. P. A. Lee and A. D. Stone, Phys. Rev. Lett. 55 (1985) 1622.

13. W. Ren, J. Wang and Z. Ma, Phys. Rev. B 72 (2005) 195407.

14. W. Ren, Z. H. Qiao, J. Wang, Q. F. Sun and H. Guo, Phys. Rev. Lett. 97 (2006) 066603.

15. J. H. Bardarson, I. Adagideli and Ph. Jacquod, Phys. Rev. Lett. 98 (2007) 196601.

16. Z. H. Qiao, J. Wang, Y. D. Wei and H. Guo, Phys. Rev. Lett. 101 (2008) 016804.

17. C. W. J. Beenakker, Rev. Mod. Phys. 69 (1997) 731 and references therein.

18. Z. H. Qiao, Y. X. Xing and J. Wang, Phys. Rev. B 81 (2010) 085114.

19. S. Feng, P. A. Lee and A. D. Stone, Phys. Rev. Lett. 56 (1986) 1960. 
20. Z. H. Qiao, W. Ren, J. Wang and H. Guo, Phys. Rev. Lett. 98 (2007) 196402.

21. Y. X. Xing, Q. F. Sun and J. Wang, Phys. Rev. B 77, 115346 (2008).

22. M. P. López-Sancho, J. M. Lópezq-Sancho and J. Rubio, J. Phys. F 14 (1984) 1205; 15 (1985) 851.

23. L. Sheng, D. N. Sheng, C. S. Ting and F. D. M. Haldane, Phys. Rev. Lett. 95 (2005) 136602 .

24. B. K. Nikolic and L. P. L. P. Zarbo, Europhys. Lett. 77 (2007) 47004.

25. E. M. Hankiewicz, L. W. Molenkamp, T. Jungwirth and J. Sinova, Phys. Rev. B 70 (2004) 241301.

26. J. Wang, B. Wang, W. Ren and H. Guo, Phys. Rev. B 74 (2006) 155307.

27. Y. G. Yao, F. Ye, X.-L. Qi, S.-C. Zhang and Z. Fang, Phys. Rev. B 75 (2007) 041401(R).

28. H. Min, J. E. Hill, N. A. Sinitsyn, B. R. Sahu, L. Kleinman and A. H. MacDonald, Phys. Rev. B 74 (2006) 165310.

29. M. König, S. Wiedmann, C. Brüne, A. Roth, H. Buhmann, L. W. Molenkamp, X.-L. Qi and S.-C. Zhang, Science 318 (2007) 766.

30. D. A. Abanin, P. A. Lee and L. S. Levitov, Phys. Rev. Lett. 96 (2006) 176803.

31. D. A. Abanin, K. S. Novoselov, U. Zeitler, P. A. Lee, A. K. Geim and L. S. Levitov, Phys. Rev. Lett. 98 (2007) 196806.

32. M. L. Mehta, Random Matrices (Academic, New York, 1991).

33. P. W. Brouwer and C. W. J. Beenakker, J. Math. Phys. 37 (1996) 4904.

34. S.-Q. Shen, Phys. Rev. B 70 (2004) 081311.

35. J. Schliemann, J. C. Egues and D. Loss, Phys. Rev. B 67 (2003) 085302.

36. Y. V. Nazarov, New J. Phys. 9 (2007) 352.

37. B. Wang, J. Wang and H. Guo, Phys. Rev. B 79 (2009) 165417.

38. M. Wimmer, I. Adagideli, S. Berber, D. Tomanek and K. Richter, Phys. Rev. Lett. 100 (2008) 177207.

39. I. Adagodeli, J. H. Bardarson and Ph. Jacquod, J. Phys.: Condens. Matter 21 (2009) 155503.

40. H. Castro Neto, F. Guinea, N. M. R. Peres, K. S. Novoselov and A. K. Geim, Rev. Mod. Phys. 81 (2009) 109 and references therein.

41. A. Varykhalov, J. Sánchez-Barriga, A. M. Shikin, C. Biswas, E. Vescovo, A. Rybkin, D. Marchenko and O. Rader, Phys. Rev. Lett. 101 (2008) 157601.

42. Y. S. Dedkov, M. Fonin, U. Rüdiger and C. Laubschat, Phys. Rev. Lett. 100 (2008) 107602.

43. O. Rader, A. Varykhalov, J. Snchez-Barriga, D. Marchenko, A. Rybkin and A. M. Shikin, Phys. Rev. Lett. 102 (2009) 057602.

44. Z. H. Qiao, S. A. Yang, W. X. Feng, W.-K. Tse, J. Ding, Y. G. Yao, J. Wang and Q. Niu, Phys. Rev. B 82 (2010) 161414.

45. F. D. M. Haldane, Phys. Rev. Lett. 61 (1988) 2015.

46. L. S. Froufe-Pérez, P. García-Mochales, P. A. Mello and J. J. Sáenz, Phys. Rev. Lett. 89 (2002) 246403.

47. X.-L. Qi, Y.-S. Wu and S.-C. Zhang, Phys. Rev. B 74 (2006) 085308. 